\title{
Biologia reprodutiva e flores de óleo em Cipura paludosa (Iridaceae)
}

\author{
Reproductive biology and oil flowers in Cipura paludosa (Iridaceae)
}

\author{
Juliana Silva dos Santos ${ }^{1}$, Sarah Maria Athiê-Souza ${ }^{1}$, Natan Messias Almeida ${ }^{3,4}$ \& Cibele Cardoso de Castro ${ }^{2}$
}

\begin{abstract}
Resumo
Cipura paludosa está inserida na família Iridaceae a qual se destaca pela grande diversidade de sistemas de polinização, ofertando recursos como néctar, pólen e óleo. Esse estudo foi conduzido em um fragmento de Mata Atlântica em área urbana, em Recife, Pernambuco. O trabalho teve como objetivo investigar a biologia floral e reprodutiva da herbácea C. paludosa. Foi avaliado o período de abertura floral, receptividade estigmática, deiscência das anteras, número de grãos de pólen e óvulos por flor, morfometria floral, experimentos de autopolinização espontânea e de polinização natural, além da observação de visitantes florais. Cipura paludosa é melitófila, autocompatível, oferece pólen e óleo (produzido em elaióforos tricomados) como recursos e recebe visitas de Plebeia sp. e Augochlora thalia. Por formar poucos frutos espontaneamente, C. paludosa se beneficia dos serviços de polinização para o seu sucesso reprodutivo. A ocorrência de abelhas não especialistas na coleta de óleo pode levar a má qualidade nos serviços de polinização, reduzindo a aptidão da espécie. Palavras-chave: Elaióforos tricomados, serviços de polinização, abelhas não especializadas, autocompatibilidade, Mata Atlântica.
\end{abstract}

\begin{abstract}
Cipura paludosa is taxonomically placed in the Iridaceae family which is notable for the diversity of pollination systems, offering resources such as nectar, pollen and oil. The study was conducted within a fragment of Atlantic Forest in Recife, Pernambuco, to investigate the floral and reproductive biology of C. paludosa. The floral opening period, stigmatic receptivity, anther dehiscense, number of pollen grains, ovules per flower and floral morphology were evaluated; experiments regarding to the reproductive system and flower visitor observations were performed. Cipura paludosa is a herbaceous plant with melittophilous and self-compatible flowers, visited by Plebeia sp. and Augochlora thalia. The flower resources produced by $C$. paludosa were pollen and oil (produced in trichomal elaiophores). To ensure reproductive success Cipura paludosa benefits from pollination services since it produces only a few fruits spontaneously. The presence of bees not specialized in oil collecting may result in poor quality pollination services consequently reducing the species reproductive output.
\end{abstract}

Key words: Trichomal elaiophores, pollination services, non-specialist bees, self- compatibility, Atlantic forest.

\section{Introdução}

Iridaceae apresenta grande diversidade de sistemas de polinização e os visitantes florais incluem diferentes espécies das ordens Hymenoptera (principalmente abelhas), Coleoptera (besouros), Diptera (moscas), Lepidoptera (borboletas e mariposas), Dermaptera e Homoptera ou aves (Nectarinidae) (Goldblatt \& Manning 2006; Vitali et al. 1995). Tais sistemas são constituídos predominantemente por animais especialistas, e a diversidade destes visitantes está relacionada à complexidade floral e aos diversos tipos de recursos florais ofertados (Goldblatt \& Manning 2006).

\footnotetext{
${ }^{1}$ Universidade Federal Rural de Pernambuco, Programa de Pós-graduação em Botânica, Depto. Biologia, Rua Dom Manoel de Medeiros s/n, Dois Irmãos, 52171-900, Recife, PE, Brasil.

${ }^{2}$ Universidade Federal Rural de Pernambuco, Unidade Acadêmica de Garanhuns, Av. Bom Pastor s/n, Boa Vista, 55292-270, Garanhuns, PE, Brasil.

${ }^{3}$ Universidade Estadual de Alagoas, Rod. AL 115, Zona Rural, Palmeira dos Índios, AL, Brasil.

${ }^{4}$ Autor para correspondência: natanmessias@yahoo.com.br
} 
Os óleos produzidos por elaióforos tricomáticos ou epiteliais, constituem o terceiro recurso mais comumente observado nas Angiospermas, ficando atrás de néctar e pólen (Buchmann 1987; Vogel 1990b; Machado 2004; Goldblatt \& Manning 2006; Alves dos Santos et al. 2007). Tal grupo de substâncias é produzido por 11 famílias de angiospermas, sendo classificadas como recurso alimentar, quando destinado à nutrição das larvas em uma mistura enriquecida com grãos de pólen, ou não-alimentar quando é utilizado na impermeabilização de ninhos (Vogel 1990a, 1990b; Michener 2000; Machado 2004; Alves dos Santos et al. 2007). Os óleos não voláteis foram registrados em Iridaceae como recurso, juntamente com o néctar e o pólen (Goldblatt \& Manning 2006).

De forma geral, flores de óleo recebem visitas de abelhas especializadas na coleta deste recurso. Estes animais, geralmente fêmeas, utilizam estruturas semelhantes a pentes, localizadas em suas pernas, para pressionar as glândulas e retirar o óleo, que é então armazenado nas escopas das pernas posteriores (Vogel 1990b; Buchmann 1987; Machado 2004; Alves dos Santos et al. 2007).

Iridaceae compreende cerca de 1800 espécies incluídas em 88 gêneros (Hilu et al. 2003) e possuem distribuição cosmopolita (Capellari Júnior 2000). A região Neotropical é considerada o segundo maior centro de diversidade, apresentando aproximadamente 30 gêneros e 250 espécies. No Brasil, foram registrados 20 gêneros e 168 espécies (Judd et al. 2009; Capellari Junior 2000). Apesar da grande representatividade do grupo, poucos são os estudos acerca da ecologia da reprodução e polinização de Iridaceae. Destacamse os estudos que avaliaram a radiação dos sistemas de polinização de Iridaceae (Goldblatt \& Manning 2006), a entomofauna visitante de Belamcanda chinensis (L.) Redouté (Vitali et al. 1995) e os recursos florais utilizados por insetos em uma comunidade campestre no sul do Brasil (Pinheiro et al. 2008).

Cipura paludosa Aubl. é uma espécie herbácea que se distribui amplamente na América tropical, desde o sul do México até o sudeste do Brasil (Goldblatt \& Henrich 1987). No Brasil tem maior ocorrência no norte, nordeste e no centrooeste, ocorrendo preferencialmente em solos arenosos sujeitos a alagamentos e a baixas altitudes, onde forma pequenas populações (Chukr 1992). Distingue-se facilmente de outras espécies de Iridaceae por suas folhas dísticas e inflorescências axilares complexas (Chukr 1992; Espejo-Serna et al. 1996; Goldblat \& Henrich 1987).

Este trabalho teve como objetivo descrever a biologia floral de Cipura paludosa, bem como analisar o comportamento dos insetos visitantes e o sistema reprodutivo da espécie.

\section{Materiais e Métodos}

Área de estudo

O estudo foi realizado no período de agosto a outubro de 2009 em um fragmento urbano de floresta atlântica do Parque Estadual de Dois Irmãos, Recife, Pernambuco, Brasil (8'30"S e $34^{\circ} 52^{\prime} 30^{\prime \prime} \mathrm{W}$ ). Com uma extensão de cerca de 370 ha, o fragmento ocupa uma área com relevo levemente ondulado e altitudes que variam de 30 a 80 m (Machado et al. 1998). O clima enquadra-se no tipo As' na classificação de Köppen (Andrade \& Lins 1965), com precipitação pluviométrica anual média em torno de $2.200 \mathrm{~mm}$ (Fidem 1987).

\section{Biologia floral}

Foram tomadas medidas das estruturas do perianto (comprimento de tépalas externas e altura do tubo formado pelas tépalas internas) e das estruturas reprodutivas (altura de estames e estigma). A duração das flores foi determinada por meio da observação dos horários de abertura e senescência das mesmas. A verificação da receptividade do estigma foi realizada através da atividade peroxidásica (Dafni 1992) e a deiscência das anteras pela observação direta das mesmas, ambas realizadas no momento da abertura das flores. O número de grãos de pólen e óvulos por flor foi obtido a partir da contagem direta sob microscopia óptica utilizando-se um contador manual. Para estimar a razão pólen/óvulo foi utilizada a metodologia de Cruden (1977). A viabilidade dos grãos de pólen foi verificada através da técnica de coloração do citoplasma pelo carmim acético (Dafni 1992). Foi avaliada a ocorrência de glândulas de odor por meio do uso de solução aquosa de vermelho-neutro (Vogel 1978). Para todas as análises realizadas foram utilizados 10 botões em pré-antese ou flores distribuídas em 10 indivíduos de duas populações.

\section{Visitantes florais}

Foram realizadas 26 horas de observações focais, durante toda a manhã, para identificar os visitantes florais, avaliar seu comportamento (contato com elementos reprodutivos, recurso 
coletado) e sua freqüência de visitas às flores. Os visitantes foram documentados com uso de registros fotográficos, capturados, montados e identificados no laboratório de Ecologia Reprodutiva de Angiospermas, no Departamento de Biologia da Universidade Federal Rural de Pernambuco.

\section{Sistema reprodutivo}

Para verificação da ocorrência de autopolinização espontânea, 26 botões em préantese foram marcados e ensacados (com sacos de "voil") durante o seu período funcional. Para verificar a formação natural de frutos, 30 flores foram marcadas e deixadas expostas à ação dos polinizadores. Para o tratamento de autopolinização espontânea e para a polinização natural foi utilizada apenas uma flor ou botão por indivíduo. Ocorrendo a formação de frutos, os mesmos foram coletados e levados ao laboratório para contabilização do número de sementes. As sementes com deformações foram consideradas abortadas e por esse motivo sem viabilidade.

\section{Análise estatística}

Foi utilizado o teste G para comparar o número de frutos e sementes formados após a autopolinização espontânea e polinização natural foram usados, respectivamente, os testes $\mathrm{G}$ e ANOVA, no programa BioEstat 5.0 (Ayres et al. 2007).

\section{Resultados}

Biologia floral

Cipura paludosa ocorre em locais úmidos e sombreados da mata do Parque Estadual de Dois Irmãos, formando pequenos agrupamentos de indivíduos, os quais emitem escapos florais e parte vegetativa relativamente inconspícua a partir de seus bulbos. Produz até quatro flores por inflorescência, as quais são subtendidas por brácteas tectrizes. Cada uma das inflorescências possui uma unidade básica do tipo ripídio, sendo que apenas uma única flor por planta abre diariamente.

As flores são hermafroditas, actinomorfas, trímeras, com perigônio mais ou menos campanulado composto por seis tépalas azuis e guias de recurso floral (óleo) de coloração amarela, dispostos na região central das tépalas internas juntamente com os elaióforos tricomados (Fig. 1). O verticilo externo diferencia-se do interno na forma e no tamanho (tépalas externas são maiores; Tab. 1), sendo que as tépalas externas são patentes e as tépalas internas são eretas e formam um tubo que envolve completamente o gineceu e androceu.

$\mathrm{O}$ androceu possui três estames férteis com anteras rimosas que produzem $4721 \pm 66$ grãos de pólen; encontram-se opostos às tépalas externas, em torno do gineceu, muito próximo e no mesmo nível do estigma (Tab. 1). As anteras estão situadas próximas aos estiletes. O gineceu possui três estiletes parcialmente unidos formando uma coluna
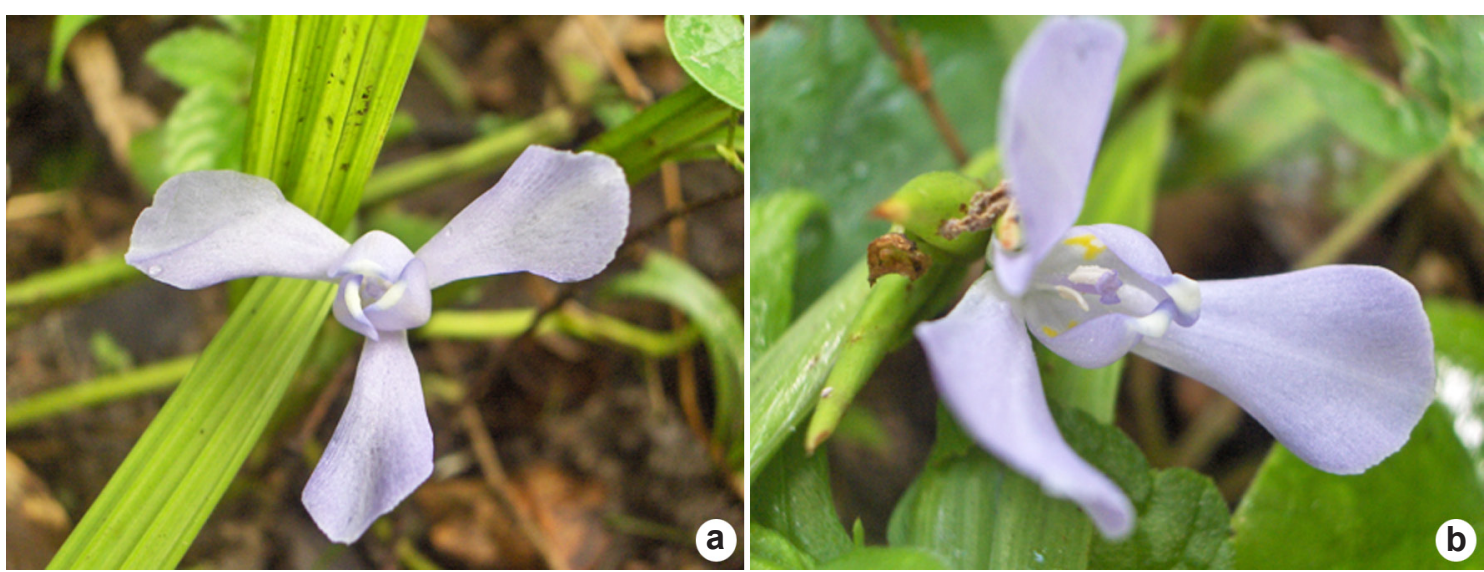

Figura 1 - Flor de Cipura paludosa em um fragmento de Floresta Atlântica de Pernambuco, nordeste do Brasil - a. flor completamente aberta, mostrando as tépalas internas (tubo) e externas (plataforma de pouso); b. detalhe dos elaióforos tricomados com guias de recurso floral.

Figure 1 - Flower of Cipura paludosa in an Atlantic Forest area in Pernambuco, northeast Brazil - a. completely open flower, showing the internal tepals (tube) and external tepals (landing platform); b. detail of trichomal elaiophores with floral resource guides. 
Tabela 1 - Dimensões das estruturas florais de Cipura paludosa em uma área de Floresta Atlântica de Pernambuco, nordeste do Brasil.

Table 1 - Size of the floral structures of Cipura paludosa in an Atlantic Forest area in Pernambuco, northeast Brazil.

\begin{tabular}{lc}
\hline Caracteres florais $(\mathbf{c m} ; \mathbf{n}=\mathbf{1 0})$ & Média (DP) \\
\hline Comprimento da tépala externa & $2,1(0,12)$ \\
Altura da tépala interna (tubo) & $1,2(0,07)$ \\
Comprimento do gineceu & $0,7(0,08)$ \\
Comprimento do estame & $0,7(0,08)$ \\
\hline
\end{tabular}

cilíndrica, bastante elaborados pela redução dos lacínios apicais e estigma truncado, ligeiramente lobado; o ovário é ínfero, tricarpelar, trilocular, pluriovulado ( $63 \pm 0,7$ óvulos) e com placentação axial. $\mathrm{O}$ fruto é uma cápsula loculicida com coloração verde durante todo o desenvolvimento, tornando-se castanho próximo à liberação das sementes.

As flores iniciam a abertura por volta das $5 \mathrm{~h}$, abrindo-se completamente por volta das $6 \mathrm{~h}$, horário em que as anteras apresentam-se deiscentes e o estigma receptivo. As flores começam a murchar por volta das $11 \mathrm{~h}$ e completam a senescência em torno de 12 h $30 \mathrm{~min}$. Após a antese, as tépalas se enrolam umas nas outras e persistem assim até o dia seguinte. As flores não exalam odor perceptível ao olfato humano, apesar de apresentarem regiões emissoras de odor no ápice das tépalas internas (tubo), na zona próxima aos guias de recurso, nos filetes e no estigma.

Cipura paludosa apresentou alta viabilidade polínica $(98 \pm 0,74 \%)$ e elevada quantidade de grãos de pólen em relação ao número de óvulos, resultando em uma razão $\mathrm{P} / \mathrm{O}$ de $75 \pm 1,1$.

Visitantes florais

Foram observadas abelhas das espécies Plebeia sp. (Apidae) e Augochlora thalia (Halictidae) visitando as flores de C. paludosa. Os visitantes coletaram pólen e o óleo das flores com picos de atividade por volta das $8 \mathrm{~h}$ da manhã, realizando uma média de duas e três visitas diárias, respectivamente. No momento da visita, pousavam nas flores utilizando as tépalas externas como plataforma de pouso, entravam completamente na flor através do tubo formado pelas tépalas internas e se movimentavam sobre as estruturas florais. Durante a visita encostavam simultaneamente a cabeça e a região ventral nas anteras e no estigma. Em Plebeia sp. o pólen era armazenado nas pernas posteriores, na região da corbícula, e em Augochlora thalia o mesmo era armazenado no ventre.

Ambas as espécies foram observadas raspando a porção interior das tépalas internas, especificamente na região dos elaióforos, efetuando a coleta do óleo e misturando-o ao pólen para armazená-lo na corbícula no caso de Plebeia sp. e no ventre no caso de $A$. thalia.

Abelhas do gênero Eufriesia e vespas do gênero Pepsis se aproximaram da planta, mas não chegaram a tocar nas estruturas reprodutivas das flores.

\section{Sistema reprodutivo}

A polinização natural resultou na maior produção de frutos comparando-se com a autopolinização espontânea $(\mathrm{G}=4.609 ; \mathrm{Gl}=1 ; \mathrm{p}=$ 0.0318; Tab. 2). O número de sementes produzidas por fruto não diferiu significativamente entre as duas condições (Tab. 2). Foi observado elevado número de sementes abortadas na autopolinização espontânea.

Tabela 2 - Resultados dos experimentos do Sistema reprodutivo em Cipura paludosa, em uma área de Floresta Atlântica em Pernambuco, nordeste do Brasil. Números seguidos pela mesma letra na coluna são estatisticamente semelhantes; Teste G (frutos) e ANOVA (sementes).

Table 2 - Results of the breeding system experiments in Cipura paludosa, in an Atlantic Forest area in Pernambuco, northeast Brazil. Numbers followed by the same letter in a column are statistically similar; Test G (fruits) and ANOVA (seeds).

\begin{tabular}{lcc}
\hline Tratamento & \% Fruto (flor) & Semente / Fruto Média (DP) \\
\hline Autopolinização espontânea & $19^{\mathrm{a}}(26)$ & $30^{\mathrm{a}}(5,4)$ \\
Polinização natural & $50^{\mathrm{b}}(30)$ & $41^{\mathrm{a}}(3,0)$ \\
\hline
\end{tabular}




\section{Discussão}

Cipura paludosa possui atributos florais supostamente atrativos às abelhas, tais como antese diurna, presença de áreas de emissão de odor, cor lilás, plataforma de pouso (tépalas), guia indicadores de recurso e centro floral diferenciado das demais partes da flor (Faegri \& Van der Pijl 1979; Biesmeijer et al. 2005). Apesar do conceito de sindromes ser discutível, e sua utilização está relacionada a possível predição da existência de grupos principais de polinizadores (Rosas-Guerrero et al. 2014 mas ver Ollerton et al. 2011), para $C$. paludosa a ocorrência de abelhas como únicos polinizadores corroboraram com as características florais observadas. O período de antese relativamente curto (7 h) de Cipura paludosa coincide com outras espécies de Iridaceae (Eggers 2008), o que pode evidenciar uma estratégia reprodutiva semelhante para estas espécies.

A produção elevada de grãos de pólen por flores justifica-se pelo fato dos visitantes florais utilizarem o pólen como recurso floral, o que diminuiria as chances da ocorrência de limitação polínica (Ashman et al. 2004). De acordo com Carmo et al. (2002), essa pode ser uma estratégia para aumentar as chances dos grãos chegarem aos estigmas, mesmo se houver grandes perdas, já que o pólen é utilizado tanto pela planta, na polinização e fecundação, quanto pelo polinizador como alimento para as larvas (Buchmann 1983; Nadia et al. 2005). Henriques (1999) afirma que espécies que fornecem pólen como recompensa floral também apresentam alta viabilidade polínica, para compensar a oferta aos visitantes, corroborando com os nossos resultados. Outro mecanismo de redução de perdas de pólen utilizado por espécies que ofertam este recurso aos visitantes (flores de pólen; Vogel 1978), é a heteranteria, caracterizada por flores que apresentam anteras com diferentes funções (alimentação e polinização) e muitas vezes diferentes tamanhos, posicionamentos e/ou coloração (Buchmann 1983; Vallejo-Marín et al. 2009). Contudo, em $C$. paludosa, as anteras, além de não apresentarem diferenciação morfológica e função, possuem cores sóbrias, não são visíveis externamente, uma vez que estão encobertas pelas tépalas internas, afastando a possibilidade do enquadramento destas flores como flores de pólen (Vogel 1978).

A presença de elaióforos tricomados caracteriza as flores produzidas por C. paludosa como flores de óleo (Vogel 1990b). A presença de estruturas glandulares unicelulares já foi relatada para essa espécie por Chauveau et al. (2012).
Flores de óleo comumente recebem visitas de abelhas especializadas na coleta deste recurso. Nos Neotrópicos os visitantes normalmente pertencem aos gêneros Centris e Epicharis, predominando abelhas solitárias do sexo feminino e de médio a grande porte (Vogel 1990b; Alves dos Santos et al. 2007; Bezerra 2008). Cipura paludosa recebeu visitas de Augochlora thalia e Plebeia sp., abelhas não especialistas na coleta de óleo, o que pode resultar em má qualidade nos serviços de polinização (Ashman et al. 2004). Contudo, estas espécies apresentaram comportamento semelhante de coleta já relatado para abelhas especialistas, que consiste em raspar os elaióforos com as pernas anteriores (Vogel 1990b; Alves dos Santos et al. 2007; Bezerra 2008). Assim, tais espécies podem ser consideradas importantes polinizadores da espécie na área de estudo, já que a formação natural de frutos foi maior do que aquela após a autopolinização. Apesar de $C$. paludosa formar frutos espontaneamente, a espécie necessita da ação dos polinizadores para elevação do seu sucesso reprodutivo. Entretanto, a ocorrência de visitantes não específicos para a coleta do principal recurso, o óleo, pode ocasionar redução do valor adaptativo pelo menor número de frutos e sementes produzidas (Schlindwein 2000).

A formação de frutos espontaneamente em Cipura paludosa deve-se à disposição das estruturas reprodutivas em suas flores o que favorece a autopolinização, em função da proximidade das anteras em relação à região estigmática. A formação de frutos após autopolinização espontânea já havia sido descrita para Belamcanda chinensis (Vitali et al. 1995), da mesma família, em taxa similar $(15,8 \%)$. Contudo, como não realizamos testes para a verificação da apomixia, não podemos descartar a ocorrência de frutos e sementes formadas por este mecanismos, ou seja sem a necessidade de fecundação.

Mesmo formando frutos espontaneamente, C. paludosa demonstrou ser preferencialmente xenógama, pelo alto valor da razão $\mathrm{P} / \mathrm{O}$ (Cruden 1977) e maior formação de frutos na polinização natural, dependendo assim da ação de visitantes para a elevação do seu sucesso reprodutivo. A formação espontânea, ainda que em baixa quantidade verificada em Cipura paludosa pode ser um indicativo de uma estratégia de segurança reprodutiva (Baker 1955, 1967). Essa mesma estratégia é normalmente reportada para espécies colonizadoras de ambientes, podendo também ser aplicada a espécies habitantes de ilhas e invasoras (Anderson et al. 2001; Rambuda \& Johnson 2004). 
Estas espécies apresentam sistemas reprodutivos simples, com a capacidade de formar frutos espontaneamente, na maioria dos casos (Baker 1955; Jaimes \& Ramirez 1999). Para C. paludosa, o potencial de colonização é elevado por ser uma espécie bulbosa com capacidade de reprodução vegetativa. Contudo, a oferta de óleo como recurso demonstra certo grau de especialização, o que pode reduzir o sucesso reprodutivo uma vez que, a espécie recebe visitas de abelhas não especialistas e pode ser o indicativo também de que embora garantida pela formação espontânea de frutos, a reprodução de C. paludosa pode receber benefícios significativos do fluxo gênico promovido pelas abelhas coletoras de óleo.

\section{Agradecimentos}

À diretoria do Parque Estadual Dois Irmãos, a autorização da pesquisa no campo.

\section{Referências}

Alves-dos-Santos, I.; Machado, I.C. \& Gaglianone, M.C. 2007. História natural das abelhas coletoras de óleo. Oecologia Brasiliensis 11: 544-557.

Anderson, G.; Bernardello, G.; Stuessy, T. F. \& Crawford D.J. 2001. Breeding system and pollination of selected plants endemic to Juan Fernández Islands. American Journal of Botany 88: 220-233.

Andrade, G.O. \& Lins, R.C. 1965. Introdução a morfoclimatologia do nordeste do Brasil. Arquivos do Instituto Ciência Terra 4: 17-28.

Ashman, T.L.; Knight, T.M.; Steets, J.A.; Amarasekare, P.; Burd, M.; Campbell, D.R.; Dudash, M.R.; Johnston, M.O.; Mazer, S.J.; Mitchell, R.J.; Morgan, M.T. \& Wilson, W.G. 2004. Pollen limitation of plant reproduction: ecological and evolutionary causes and consequences. Ecology 85: 2408-2421.

Ayres, M.; Ayres Jr., M.; Ayres, D.L. \& Santos, A.S.S. 2007. Biostat: aplicações estatísticas nas áreas das ciências biomédicas. Sociedade Cívil Mamirauá, Belém. 364p.

Baker, H.G. 1955. Self-compatibility and establishment after "long-distance" dispersal. Evolution 9: 347-349.

Baker. H.G. 1967. Support for Baker's Law-as a rule. Evolution 21: 853-856.

Bezerra, E.L.S. 2008. Guilda de flores de óleo do Parque Nacional do Catimbau: fenologia, polinização e sistema reprodutivo. Tese de Doutorado. Universidade Federal de Pernambuco, Recife. 173p.

Biesmeijer, J.C.; Giurfa, M.; Koedam, D.; Potts, S.G.; Joel, D.M. \& Dafni, A. 2005 Convergent evolution: floral guides, stingless bee nest entrances, and insectivorous pitchers. Naturwissenschaften 92: 444-450
Buchman, S.L. 1983. Buzz pollination in angiosperms. In: Jones, C.E. \& Little, R.J. (eds.). Handbook of Experimental Pollination. Van Nostrand Reinhold, New York. Pp. 77-113.

Buchmann, S.L. 1987. The ecology of oil flowers and their bees. Annual Review of Ecology and Systematics 18: 343-369.

Capellari Jr., L. 2002. Iridacées Brésiliennes. França. Iris et Bulbeuses 144: 22-25.

Carmo, R.M. \& Franceschinelli, E.V. 2002. Polinização e biologia floral de Clusia arrudae Planchon \& Triana (Clusiaceae) na Serra da Calçada, município de Brumadinho, MG. Revista Brasileira de Botânica 25: 351-360.

Chauveau, O.; Eggers, L.; Souza-Chies, T.T. \& Nadot, S. 2012. Oil-producing flowers within the Iridoideae (Iridaceae): evolutionary trends in the flowers of the New World genera. Annals of Botany 110: 713-729.

Chukr, N.S. 1992. Novos taxons em Iridaceae da Serra do Cipó (Minas Gerais - Brasil). Bobti Botânica, Unh 73: 103-109.

Cruden, R.W. 1977. Pollen-ovule ratios: a conservative indicator of breeding systems in flowering plants. Evolution 31: 32-46.

Dafni, A. 1992. Pollination Ecology - a practical approach. Oxford University Press, Oxford. 250p.

Eggers, L. 2008. A família Iridaceae no Parque Estadual de Itapuã, Viamão, Rio Grande do Sul, Brasil. Revista Brasileira de Biociências 6: 167-175.

Esperjo-Sena, A. \& Lopez-Ferrari, A.R. 1996. Comentários floristico-ecologicos sobre las Iridaceas mexicanas. Acta Botanica Mexicana 34: $25-47$.

Faegri, K. \& Pijl, L. 1979. The principles of pollination ecology. Pergamon Press, London. 248p.

FIDEM - Fundação de Desenvolvimento da Região Metropolitana do Recife. 1987. Região metropolitana do Recife: reservas ecológicas. Série de Desenvolvimento Urbano e Meio Ambiente. $108 \mathrm{p}$.

Goldblatt, P. \& Henrich, J.E. 1987. Notes on Cipura (Iridaceae) in South and Central America, and a new species from Venezuela. Annals of the Missouri Botanical Garden 74: 333-340.

Goldblatt, P. \& Manning, J.C. 2006. Radiation of pollination systems in the Iridaceae of sub-Saharan Africa. Annals of Botany 97: 317-344.

Henriques, R.P.B. 1999. Ecologia da polinização de Oatea hexasperma (St. Hil.) Baill. (Ochnaceae) em cerrado no Brasil Central. Boletim do Herbário Ezechias Paulo Heringer 4: 46-64.

Hilu, K.; Borsch, T.; Muller K.; Soltis D.; Soltis, Ps; Savolainen, V. \& Chase, M.W. 2003. Angiosperm phylogeny based on matK sequence information. American Journal of Botany 90: 1758-1776. 
Jaimes, I. \& Ramirez, N. 1999. Breeding systems in a secondary deciduous forest in Venezuela: the importance of life form, habitat, and pollination specificity. Plant Systematics and Evolution 215: 23-36.

Judd, W.S.; Campbell, C.S.; Kellogg, E.A.; Stevens, P.F. \& Donoghue, M.J. 2009. Sistemática vegetal: um enfoque filogenético. $3^{\mathrm{a}}$ ed. Artmed, Porto Alegre. 612p.

Machado, I.C.; Lopes, A.V. \& Pôrto, K.C. 1998. Reserva ecológica de Dois Irmãos: estudos em um remanescente de Mata Atlântica em área urbana. SECTMA, Editora Universitária da UFPE, Recife. 326p.

Machado, I.C. 2004. Oil-collecting bees and related plants: a review of the studies in the last twenty years and case histories of plants occurring in NE Brazil. In: Freitas, B.M. \& Pereira, J.O.P. Solitary bees. Conservation, rearing and management for pollination. Imprensa Universitária, Fortaleza. Pp. 255-280.

Michener, C.D. 2000. The bees of the world. Johns Hopkins University Press, Baltimore. 913p.

Nadia, T.L. \& Machado, I.C. 2005. Polinização por vibração e sistema reprodutivo de duas espécies de Sauvagesia L. (Ochnaceae). Revista Brasileira de Botânica 28: 255-265.

Pinheiro, M.; Abrão, B.E.; Harter-Marques, B. \& Miotto, S.T.S. 2008. Floral resources used by insects in a grassland community in Southern Brazil. Revista Brasileira de Botânica 31: 469-489.

Rambuda, T.D. \& Johnson, S.D. 2004. Breeding systems of invasive alien plants in South
Africa: does Baker's rule apply? Diversity and Distributions 10: 409-416.

Radford, A.E.; Dickinson, W.C.; Massey, J.R. \& Bell, C.R. 1974. Vascular plant systematics. Harper \& Row Publishers, New York. 891p.

Rosas-Guerrero, V.; Aguilar, R.; Martén-Rodríguez, S.; Ashworth, L.; Lopezaraiza-Mikel, M.; Bastida, J. M. \& Quesada, M. 2014. A quantitative review of pollination syndromes: do floral traits predict effective pollinators? Ecology letters 17: 388-400.

Schlindwein, C. 2000. A importância de abelhas especializadas na polinização de plantas nativas e conservação do meio ambiente. Anais do Encontro sobre Abelhas 4: 131-141.

Vallejo-Marín, M.; Manson, J.S.; Thomson, J.D. \& Barrett, S.C.H. 2009. Division of labour within flowers: heteranthery, a floral strategy to reconcile contrasting pollen fates. Journal of Evolutionary Biology 22: 828-839.

Vitali, M.J.; Dutra, J.C.S. \& Machado, V.L.L. 1995. Entomofauna visitante de Belamcanda chinensi (L.) DC. (Iridaceae) durante o período de floração. Revista Brasileira de Zoologia 12: 239-250.

Vogel, S. 1978. Evolutionary shifts from reward to deception in pollen flowers. In: Richards, A.J. (ed.). The pollination of flowers by insects. Academic Press, London. Pp. 89-96.

Vogel, S. 1990a. The role of scent glands in pollination. A.A. Balkema, Rotterdam, Netherlands. 202p.

Vogel, S. 1990b. History of the Malpighiaceae in the light of pollination ecology. Memoirs of the New York Botanical Garden 55: 130-142. 